\title{
Change of intestinal microbiota in cerebral ischemic stroke patients
}

\author{
$\mathrm{Na} \mathrm{Li}^{1 \dagger}$, Xingcui Wang ${ }^{2 \dagger}$, Congcong Sun ${ }^{3 \dagger}$, Xinwei $\mathrm{Wu}^{4+}$, Mei $\mathrm{Lu}^{4}$, Youfeng $\mathrm{Si}^{5}$, Xiang Ye ${ }^{4}$, Tan Wang ${ }^{4}$, Xiaolin $\mathrm{Yu}^{4}$,
} Xinjing Zhao ${ }^{4}$, Na Wei ${ }^{4}$ and Xingbang Wang ${ }^{4 *}$

\begin{abstract}
Background: Gut microbiota has been suggested to play a role in stroke patients. Nevertheless, little is known about gut microbiota and the clinical indexes in stroke patients.

Methods: Total of 30 cerebral ischemic stroke (Cl) patients and 30 healthy control were enrolled in this study and the fecal gut microbiota was profiled via Illumina sequencing of the $16 \mathrm{~S}$ rRNA V1-V2. The National Institutes of Health Stroke Scale (NIHSS) were used to quantify stroke severity and modified Rankin scale (mRS) to assess outcome for $\mathrm{Cl}$ patients. The correlations between the clinical indexes and microbiota were evaluated.

Results: Though the microbial a-diversity and structure is similar between $\mathrm{Cl}$ patients and healthy controls, the gut microbiota of $\mathrm{Cl}$ patients had more short chain fatty acids producer including Odoribacter, Akkermansia, Ruminococcaceae_UCG_005 and Victivallis. We also found that the special microbes were correlation with serum index, such as norank_O__Mollicutes_RF9, Enterobacter, Ruminococcaceae_UCG-002 were negative correlation with LDL $(r=-0.401, P<0.01), \operatorname{HDL}(r=-0.425, P<0.01)$ and blood glucose $(r=-0.439, P<0.001)$, while the HDL was significantly positive correlation with the genus Ruminococcus_1 $(r=0.443, P<0.001)$. The Christensenellaceae_R-7_ group and norank_f_Ruminococcaceae was significantly positive correlation with NIHSS1M $(r=0.514, P<0.05$; $r=0.449, P<0.05)$ and $\mathrm{mRS}(r=0.471, P<0.05, r=0.503, P<0.01)$, respectively. On the other hand, the genus Enterobacter was significantly negative correlation with NIHSS1M $(r=0.449, P<0.05)$ and $\mathrm{mRS}(r=0.503, P<0.01)$.

Conclusions: This study suggests that $\mathrm{Cl}$ patients showed significant dysbiosis of the gut microbiota with enriched short chain fatty acids producer, including Odoribacter, Akkermansia. This dysbiosis was correlation with the outcomes and deserves further study.
\end{abstract}

Keywords: Cerebral ischemic stroke, Microbiota, Dysbiosis, Outcomes

\section{Background}

Cerebrovascular accidents (stroke) is brain injury caused by disruption the supply of blood to a brain region, which could result in permanent neurological deficits or death [1]. Now, stroke is global health problem and now became to the second leading cause of death as well as the third leading cause of disability [2]. Basing on the underlying pathology, the stroke was classified to ischemic and hemorrhagic stroke [2]. The cerebral Ischemic

\footnotetext{
* Correspondence: 20893792@qq.com

${ }^{\dagger} \mathrm{Na}$ Li, Xingcui Wang, Congcong Sun and Xinwei Wu contributed equally to this work.

${ }^{4}$ Department of Geriatric Medicine, Qilu Hospital of Shandong University; Key Laboratory of Cardiovascular Proteomics of Shandong Province, Qilu Hospital of Shandong University, Jinan 250012, China

Full list of author information is available at the end of the article
}

stroke $(\mathrm{CI})$ is responsible for $85 \%$ of all strokes, and the hemorrhagic stroke accounts for about the rest 15\% [2]. The most ischemic stroke is caused by the middle cerebral artery occlusion, resulting in the brain tissue damage in the affected territory, which is followed by inflammatory and immune response [1]. Despite the stroke debilitated the neurological deficits, infection is the superior cause of death after stroke [3]. It was found that $90 \%$ of stroke cases may be correlated with behavioral factors including poor diet, smoking, and low physical activity as well as metabolic factors including obesity, hypertension, diabetes mellitus. Previous study also found that gut microbiota might be as a risk factor for stroke [4].

(c) The Author(s). 2019 Open Access This article is distributed under the terms of the Creative Commons Attribution 4.0 International License (http://creativecommons.org/licenses/by/4.0/), which permits unrestricted use, distribution, and reproduction in any medium, provided you give appropriate credit to the original author(s) and the source, provide a link to the Creative Commons license, and indicate if changes were made. The Creative Commons Public Domain Dedication waiver (http://creativecommons.org/publicdomain/zero/1.0/) applies to the data made available in this article, unless otherwise stated. 
The gastrointestinal tract is thought to be a major immune organ which was equipped with the largest pool of immune cells, accounting for more than $70 \%$ of the entire immune system. More and more evidences propose that the gut inflammatory along with immune response plays an essential role in the pathophysiology of stroke and this may become an important therapeutic target for treatment of stroke [5]. It was found that gut microbiota plays a vital role in regulation of the immune system. The gut microbiota was also revealed to be an important influence factor for stroke in a mouse model [6-9]. In addition, stroke usually cause gut dysmotility, gut microbiota dysbiosis, gut hemorrhage, as well as gut-origin sepsis, resulting in poor prognosis. However, there are little about the characteristics of gut microbiota from stroke patient [10].

This study aims to determine the characteristic of gut microbiota of stroke patient. We also evaluated the relations between gut microbiota and serum indexes or outcome by Spearman's rank correlation.

\section{Results}

\section{Subjects}

Total of 60 subjects, including $30 \mathrm{CI}(21$ males, 9 females) and 30 healthy control (18 males, 12 females) were recruited in this study (Table 1). It was found that there were no significant differences in gender (male: 70 . $\%$ vs. $60.0 \%, P=0.42)$ and age $(60.47 \pm 10.57$ vs. $64.17 \pm$ $12.67, P=0.31$ ) between the CI group and healthy control group. The LDL, GLU, UA, TG, HCY of CI patients were similar with the healthy control. The HDL of CI patients was lower than that of healthy control group $(1.10 \pm 0.19$ vs. $1.29 \pm 0.24, \quad P<0.01) \quad$ (Table 1$)$. The NIHSS at $7 \mathrm{~d}$ (NIHSS $1 \mathrm{~W}$ ) and 1 month (NIHSS $1 \mathrm{M}$ ), modulate RANK score (mRS) and serum index of each subjects were shown in Additional file 1: Table S1 and Additional file 2: Table S2.

Table 1 Characteristics of the study participants

\begin{tabular}{llll}
\hline & $\mathrm{Cl}(n=30)$ & $\mathrm{HC}(\mathrm{n}=30)$ & $P$ \\
\hline Sex (male, \%) & $21(70 \%)$ & $18(60 \%)$ & 0.42 \\
Age & $60.47 \pm 10.57$ & $64.17 \pm 12.67$ & 0.31 \\
LDL & $2.20 \pm 0.68$ & $2.30 \pm 0.50$ & 0.51 \\
HDL & $1.10 \pm 0.19$ & $1.29 \pm 0.24$ & $<0.01$ \\
UA & $303.8 \pm 64.5$ & $282.3 \pm 62.7$ & 0.20 \\
GLU & $4.92 \pm 0.67$ & $5.20 \pm 0.76$ & 0.14 \\
HCY & $12.94 \pm 3.93$ & $12.36 \pm 3.33$ & 0.54 \\
TG & $1.24 \pm 0.47$ & $1.33 \pm 0.31$ & 0.39 \\
\hline
\end{tabular}

$H D L$ high-density lipoprotein, $L D L$ low-density lipoprotein, GLU glucose of blood, UA uric acid, TG triglycerides, $\mathrm{HCY}$ homocysteine
The microbial a-diversity and structure is similar between $\mathrm{Cl}$ and $\mathrm{HC}$ group

We obtained a total of 2,191,980 high-quality sequences by quality-filtering with a coverage above $99.0 \%$. After removing rare OTUs., 19,869 sequences per sample were obtained which were clustered to 955 OTUs. The results of bacterial community richness and diversity were shown in Table 2. It was found that there was no difference in shannon, simpson, ace or chao index between two group.

The similarity of the bacterial community structures between $\mathrm{CI}$ group and healthy control group was evaluated by PCoA (Fig. 1). There was no obvious difference of the microbiota structure between the 2 groups. Then, ANOSIM were performed and also found a high similarity in the bacterial community between CI patients and the healthy control based on two algorithms (unweighted unifrac, $\mathrm{r}=0.0254, P=0.132$; weighted unifrac $\mathrm{r}=-0.012, P=0.649$, respectively).

\section{Differentially abundant taxon in microbiota of $\mathrm{Cl}$ patients and healthy control}

Most of the gut bacteria detected in this study falls into 3 phyla: Bacteroidetes, Firmicutes, and Proteobacteria (Fig. 2a). The main genus of gut micriobiota (the percentages were above $1 \%$ ) includes 24 genera and they compose up to $80 \%$ of the total microbiota, such as Bacteroides, Prevotella, Faecalibacterium, Escherichia/Shigella, Phascolarctobacterium, and Roseburia (Fig. 2b). The taxa that most likely reveal the differences between $\mathrm{CI}$ and healthy control group were identified by LEfSe (Fig. 3). At the genus level, Anaerostipes and Ruminiclostridium_5 were significantly enriched in the healthy group, while Odoribacter, Akkermansia, Ruminococcaceae_UCG_005, norank_p_Flavobacteriaceae, norank_p_Parcubacteria, and Victivallis exhibited relatively higher abundance in the CI group (Fig. 3a). We also observed that the genus Enterobacter, Pyramidobacter, and Lachnospiraceae UCG_001 increased in mild CI patients (NIHSS score $\leq$ 4) (Fig. 3b). While, genus Ruminococcaceae_UCG-002, Christensenellaceae_R-7_group, Ruminococcaceae_UCG005 and norank_f_Ruminococcaceae increased in severe stroke patients (NIHSS score > 4) (Fig. 3b).

\section{The gut microbiota was related to the clinical indices}

The serum indices of each subjects were shown in Additional file 1: Table S1. We try to find relationship

Table 2 The index of a-diversity

\begin{tabular}{llll}
\hline & $\mathrm{Cl}(n=30)$ & $\mathrm{HC}(n=30)$ & $P$ \\
\hline Shannon & $3.69 \pm 0.52$ & $3.59 \pm 0.59$ & 0.3515 \\
Simpson & $0.09 \pm 0.06$ & $0.10 \pm 0.07$ & 0.4786 \\
Ace & $555.68 \pm 51.24$ & $552.19 \pm 43.47$ & 0.4679 \\
Chao & $565.64 \pm 55.61$ & $558.79 \pm 48.05$ & 0.7775 \\
\hline
\end{tabular}




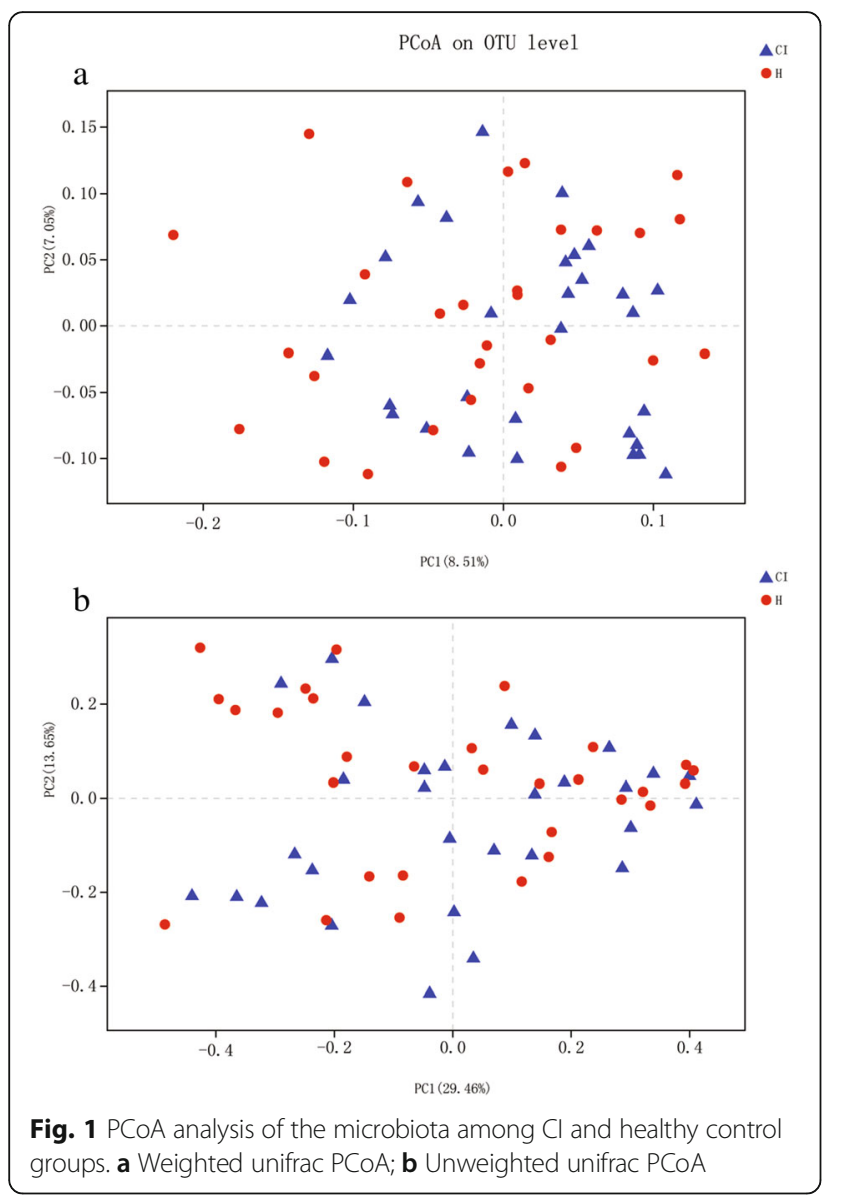

between the gut microbiota and the serum indices including TG, LDL, HDL, UA, Glu, and Hcy by Spearman's rank correlation coefficient. We found strong correlations $(r>0.22$ or $<-0.22, P<0.05)$ between them as shown in Fig. 4. A significant positive correlation was exhibited between LDL and genus Bacteroides $(\mathrm{r}=0.42$, $P<0.01)$ as well as [Eubocterium]_rectole_group $(\mathrm{r}=$ $0.336, P<0.01)$. While, it was found that norank_O_ _Mollicutes_RF9 was negative correlation with LDL ( $\mathrm{r}=$ - 0.401, $P<0.01)$. The HDL was significantly positive correlation with the genus Ruminococcus_1 $(\mathrm{r}=0.443$, $P<0.001)$, Ruminococcus_2 $(\mathrm{r}=0.381, \quad P<0.01)$ and Lachnospiraceae_NK4A136_group $(\mathrm{r}=0.338, \quad P<0.01)$ and negative correlation with the genus Enterobacter $(\mathrm{r}=-0.425, P<0.01)$. The UA was found positive correlation with Dialister $(r=0.279, P<0.05)$ and the GLU was found significantly negative correlation with Ruminococcaceae_UCG-002 $(\mathrm{r}=-0.439, P<0.001)$, Alistipes $(\mathrm{r}=-0.313, P<0.05)$, and Ruminococcus_1 $(\mathrm{r}=-0.287$, $P<0.05)$. And the Hcy was found positive correlation with Megamonas $(\mathrm{r}=0.291, P<0.05)$ and Fusobacterium $(\mathrm{r}=0.297, P<0.05)$.

The gut microbiota was correlated with the severity and outcome of $\mathrm{Cl}$

The severity and outcome of CI patients could be evaluated by NIHSS and mRS (Additional file 2: Table S2). Then, we do the Spearman's rank correlation coefficient analysis and attempt to explore the relationship between the gut microbiota and severity or outcome including NIHSS $1 \mathrm{~W}$ NIHSS $1 \mathrm{M}$ and mRS (Fig. 5). As a result, the genus Christensenellaceae_R-7_group was significantly positive correlation with NIHSS1W $(\mathrm{r}=0.488, P<0.01)$, NIHSS1M $(r=0.514, P<0.05)$ and mRS $(r=0.471, P<$ 0.05). While, the genus norank_f_Ruminococcaceae was also significantly positive correlation with NIHSS1M $(r=0.449, P<0.05)$ and $\mathrm{mRS}(r=0.503, P<0.01)$. In contrast, the genus Enterobacter was significantly positive correlation with NIHSS1M $(r=0.449, \quad P<$ $0.05)$ and $\mathrm{mRS}(r=0.503, P<0.01)$.
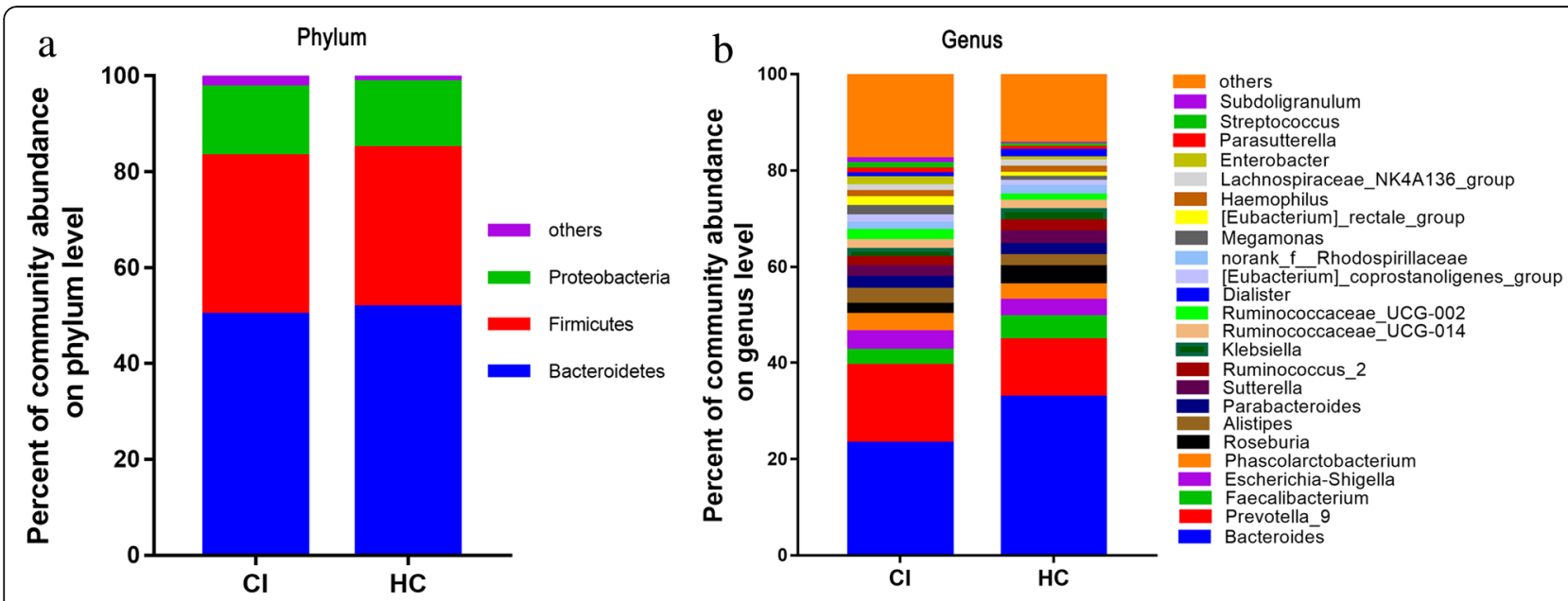

Fig. 2 The relative taxa abundance between $\mathrm{Cl}$ and healthy control groups. a Phylum; b Genus 


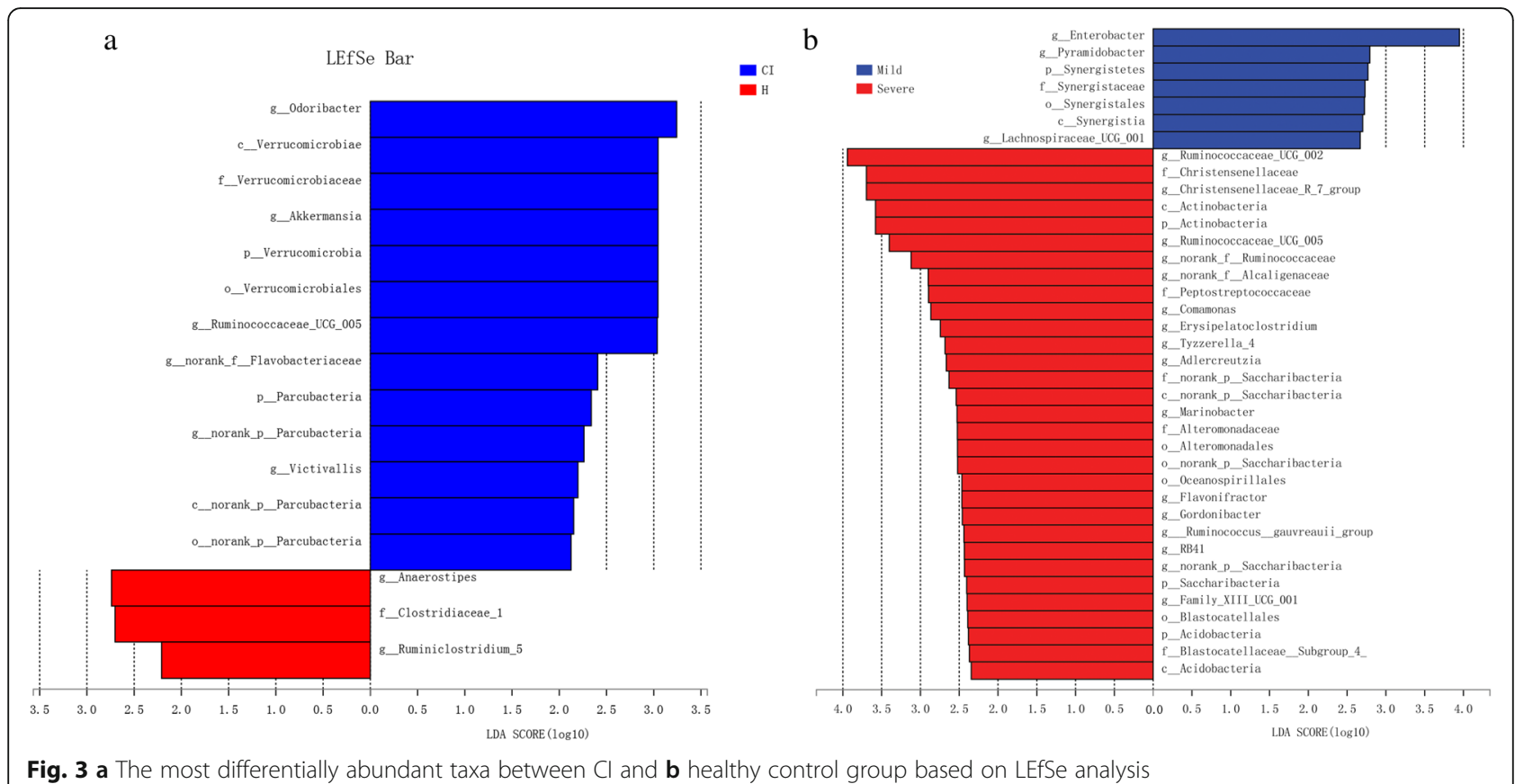

Fig. 3 a The most differentially abundant taxa between $\mathrm{Cl}$ and $\mathbf{b}$ healthy control group based on LEfSe analysis

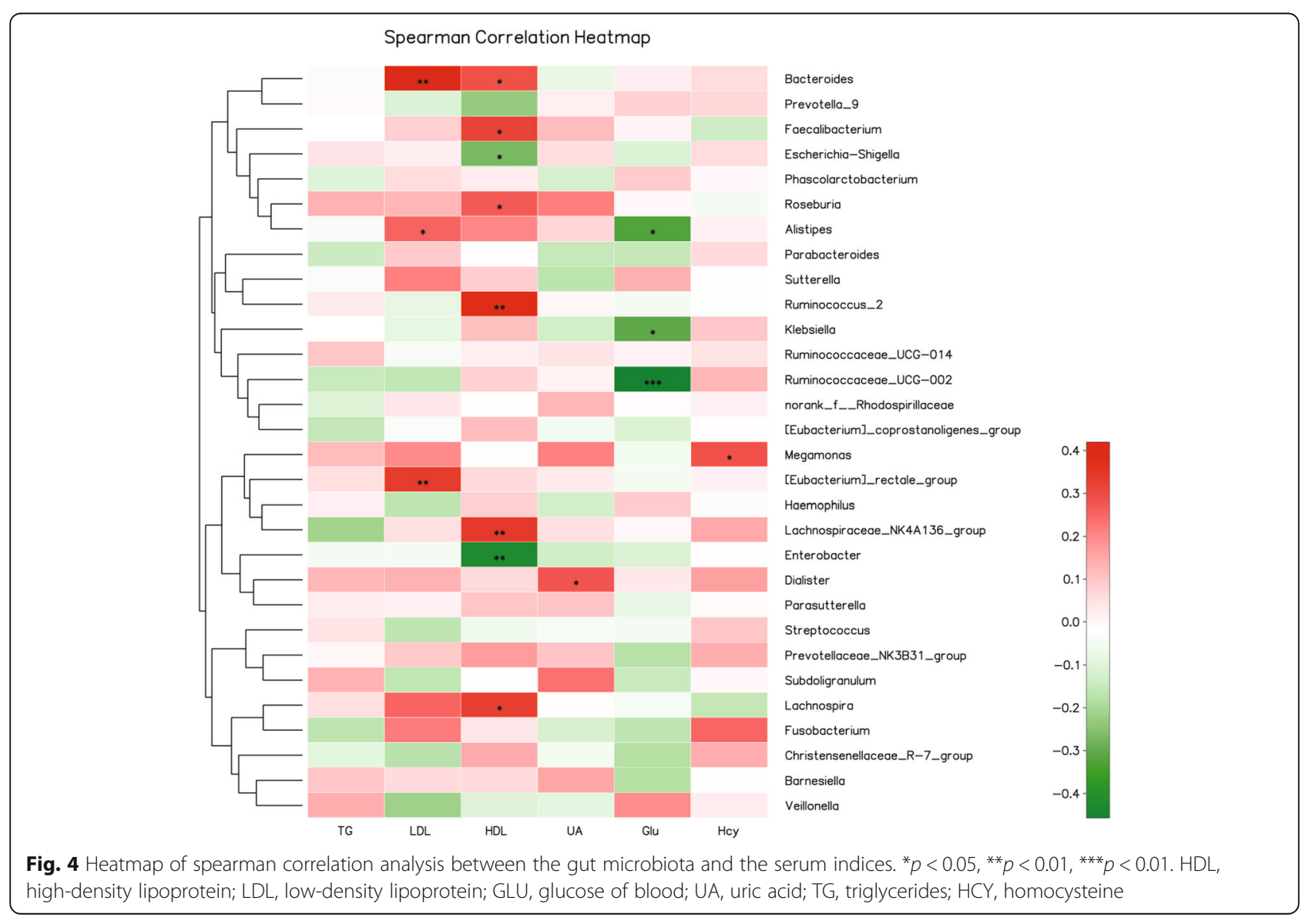




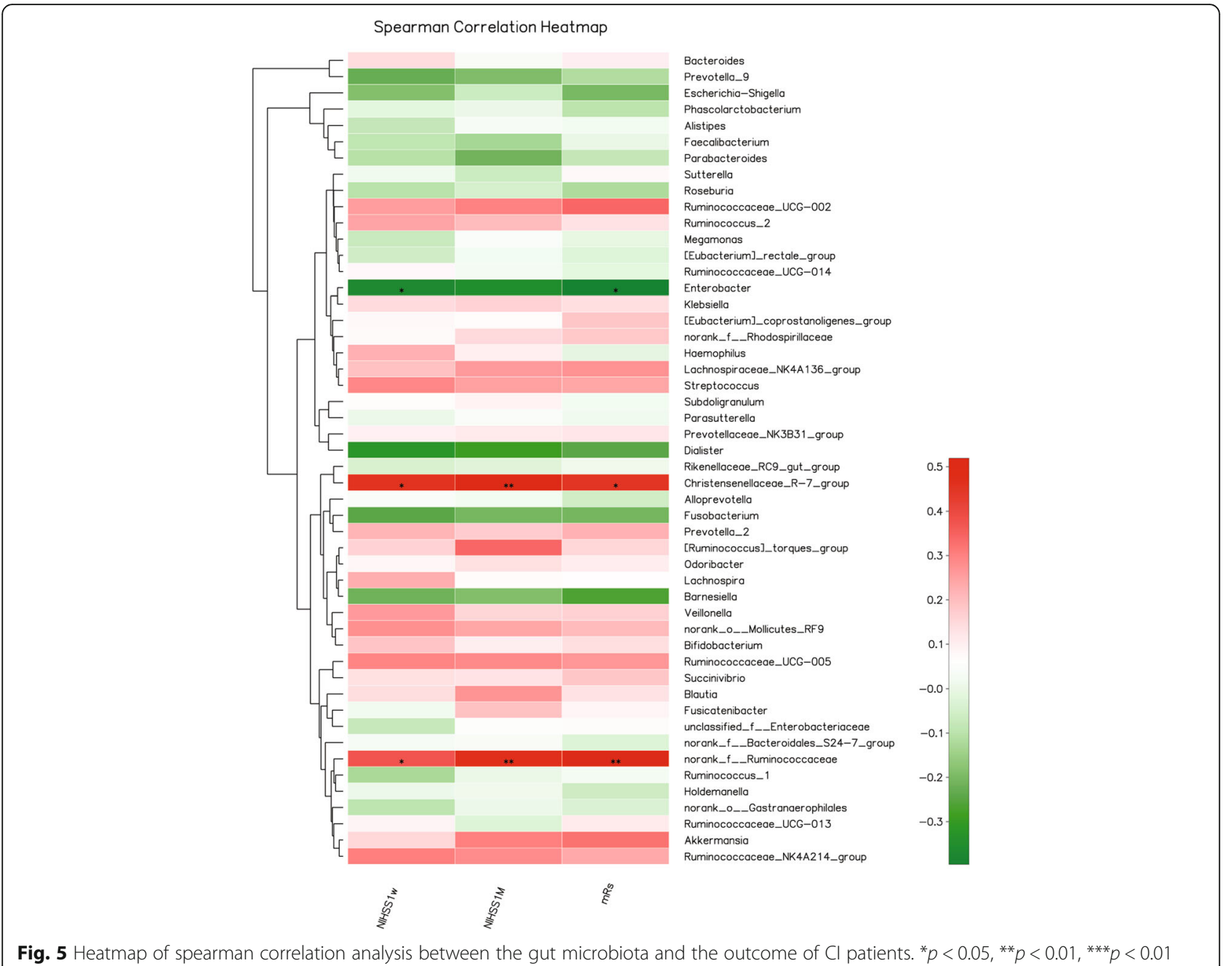

\section{Discussion}

In this study, the results demonstrate that certain changes in fecal microbiota was happened in ischemic stroke patients, such as the increasement short-chain fatty acids (SCFAs)-producing taxonomies Odoribacter and Akkermansia. Furthermore, the levels the genus Christensenellaceae_R-7_group, norank_f_Ruminococcaceae, and Enterobacter were correlated with the severity, while the level of genus Christensenellaceae_R-7_group was positively correlated with the outcome of CI patients. In addition, we also found that the microbiota was correlated with the human hematological index.

Previous studies have proved that gut microbiota play a vital role in stroke. It was reported that depletion of gut bacteria via antibiotic administration decreased the survival rate of mice following the induction of ischemia [11]. In mice, Houlden et al., found that the composition of caecal microbiota was altered by experimental stroke, such as specific changes of Peptococcaceae and Prevotellaceace [6]. Benakis et al. found that the microbiota dysbiosis would affect the ischemic stroke outcome via suppressing the traffick of effector $\mathrm{T}$ cells from the gut to the leptomeninges in middle cerebral artery occlusion model [9]. Though only a few studies have investigated the role of microbiota in stroke patient, dysregulation of the microbiota in patients following stroke have been identified [12-14]. Yin et al., found that ischemic stroke was associated with different microbiota structure and more opportunistic pathogens, such as Megasphaera, Enterobacter, Oscillibacter, along with fewer commensal or beneficial genera such as Prevotella, Bacteroides, and Faecalibacterium [13]. After that, Yamashiro et al., found that ischemic stroke was independently associated with increasement of Atopobium cluster and Lactobacillus ruminis, and decreased numbers of $L$. sakei subgroup based on qRT-PCR [12]. In another study, gut microbiota dysbiosis was identified with increased abundance of Escherichia, Bacteroides, Megamonas, Parabacteroides, and Ruminococcus in the CI and IS patients compared with the healthy group. In the higher risk of 
stroke people, there was no significant change in alpha diversity index of the microbiota. However, enrichment of opportunistic pathogens together with low abundance of butyrate-producing bacteria were found in subjects with high risk of stroke [4]. In this study, we also found that the microbial $\alpha$-diversity and structure was similar between CI patients and healthy control. However, the Anaerostipes and Ruminiclostridium_5 were significantly enriched in the healthy group, while Odoribacter, Akkermansia, Ruminococcaceae_UCG_005, norank_p_Flavobacteriaceae, and Victivallis represented a relatively higher abundance in CI group (Fig. 2a). Of them, Odoribacter, Akkermansia, Ruminococcaceae_UCG_005 and Victivallis are known producer of SCFAs, including acetate, propionate, and butyrate [4, 15-17]. Meanwhile, significant differences within the $\mathrm{CI}$ group was also observed that the Enterobacter, Pyramidobacter, and Lachnospiraceae_UCG_001 increased in mild CI patients (NIHSS score $\leq 4)$. While, genus Ruminococcaceae_UCG002, Christensenellaceae_R-7_group, Ruminococcaceae_ UCG-005 and norank_f_Ruminococcaceae increased in severe stroke patients (NIHSS score $>4$ ) (Fig. 2b). These results suggest that the intestinal flora may have been involved in the human stroke and correlated to the severity of CI.

SCFAs are a major class of bacterial metabolites obtained from fermentation of otherwise indigestible polysaccharides (fibers) in the colon by bacteria [18]. Preview studies indicated that SCFAs have important role in maintaining the intestinal integrity and anti-inflammatory. As a result, beneficial effects of SCFAs have been found in several disease models, such as colitis, metabolic syndrome and so on [19]. In this study, we found that short-chain fatty acids (SCFAs)-producing taxonomies Odoribacter and Akkermansia increased in CI patient. Akkermansia muciniphila is the single species of genus Akkermansia in the human which accounts $1-3 \%$ of the total bacterial cells in feces [20]. It was found that $A$. muciniphila was inversely associated with diabetes, obesity, cardiometabolic diseases and low-grade inflammation [16]. Previous study demonstrated that the shift in mucosal microbial composition induced by the stroke were an increasement of $A$. muciniphila along with an excessive abundance of Clostridial species in mice [8]. However, it was reported that Akkermansia decreased in CI patient [14]. The Akkermansia was of $20 \%$ abundance of the total microbe in one normal control and this might cause statistical bias. In this study, we found that Akkermansia significantly increased in CI patient $(P<0.05)$ (Fig. $2 \mathrm{c})$. It was reported that $A$. muciniphila could use mucin to produce high levels acetate which may be consumed by butyrate-producing Ruminococcaceae to promote butyrate production [4]. In this study, genus Odoribacter, a butyrate producer which belongs to phylum Bacteroidetes [21], also increase in CI patient. This indicated that the simultaneous increase of these two bacteria might promote the production of butyrate. Butyrate is a preferential energy source for epithelial cells and it preserves the epithelial heath [22]. Butyrate also could impact the expression level of genes which are aroused by $A$. muciniphila in epithelial cells [23]. Therefore, Stanley et al. thought that the Akkermansia may play a crucial role in healing of wound damage via promotion the butyrate levels, resulting in consolidate epithelial integrity in the post-stroke mice [8]. In addition, $A$. muciniphila could induce mucus production and expression of Reg3y in colon, resulting microbiota remodeling [24]. Thus, further work needs to be done to find out the possible role of Akkermansia and Odoribacter in post-stroke.

Regarding the sequelae of a stroke, predicting the severity and outcome is important to $\mathrm{CI}$ patients and their families in guiding the treatment [25]. Many biomarkers offer the potential to predict the outcome of stroke, including TG/HDL-C [26], IL-6, NT-proBNP [27], and serum YKL-40 [28]. In this study, the genus Christensenellaceae_R-7_group and norank_f_Ruminococcaceae was significantly positive correlation with NIHSS1M $(\mathrm{r}=0.514, P<0.05 ; \mathrm{r}=0.449, P<0.05)$ and $\mathrm{mRS}(\mathrm{r}=$ 0.471, $P<0.05, \mathrm{r}=0.503, P<0.01$ ), respectively. On the other hand, the genus Enterobacter was significantly negative correlation with NIHSS1M $(\mathrm{r}=0.449, P<0.05)$ and $\mathrm{mRS}(\mathrm{r}=0.503, P<0.01)$. Yin et al. found that opportunistic pathogens including Enterobacter enriched in stroke patients [13]. However, the relationship between genus Enterobacter and the severity or outcome is unknown. We also found that the genus Enterobacter was negative correlation with the HDL-C $(r=-0.425$, $P<0.01$ ), which was considered as a potential protective factor against CVD [29]. Thus, these results suggested that the HDL-C was positive correlation with the NIHSS at 1 month $(\mathrm{r}=0.449, P<0.05)$ and $\mathrm{mRS}$ and this is inconsistent with previous studies [30]. On the other hand, we also found that the genus Enterobacter increased in mild CI patients (NIHSS score $\leq 4)$. These patients usually have a better outcome than severe CI patients. These might be the reason that the genus Enterobacter was negative correlation with NIHSS $1 \mathrm{M}$ and mRS. Until now, we knew little about the genus of Christensenellaceae_R-7_group and norank_f_Ruminococcaceae. Further study to identify the potent microbial biomarkers for predicting the outcome of stroke patients need to be done.

\section{Conclusion}

This study suggests that dysbiosis of the gut microbiota in CI patients with enriched SCFAs producer, including Odoribacter, Akkermansia. This dysbiosis was correlation 
with the outcomes and might be used as biomarkers for the long-term outcome which clearly deserves further study.

\section{Methods Subjects}

Total of $30 \mathrm{CI}$ patients were selected. They were recruited from Qilu Hospital (Jinan, China) between May 2017 and January 2018, and had been diagnosed by skull computed tomography examination. The subjects enrolled in this study received and written the informed consent. The National Institutes of Health Stroke Scale (NIHSS) > 4 was defined as severe stroke. In addition, 30 healthy volunteers who were examined to ensure that they had no metabolic, cardiovascular or cerebrovascular diseases or cancer were selected as the normal group. All these individuals did not receive antibiotics or probiotic before 4 weeks prior to the specimen collection. This study was approved by the Ethical Committee of the Qilu Hospital of Shandong University (Jinan, China).

\section{Sample collection and 16S rRNA sequencing}

The first available fecal samples of CI patients after admission (within $48 \mathrm{~h}$ ) were collected. The samples and its genomic DNA were immediately frozen in liquid nitrogen and then stored at $-80^{\circ} \mathrm{C}$. Genomic DNA of microbes in fecal samples was extracted by CTAB assay. About $1 \mathrm{mg}$ of each sample was used for DNA quantification by a NanoDrop ND-1000 spectrophotometer (NanoDrop Technologies, Wilmington, DE, USA).

For analyzing the microbial populations, amplification of the V1-V2 region of the 16S rRNA gene was performed via barcode-indexed primers 27F: 5' AGAGTTTGATCM TGGCTC G, 338R-I 5' GCWGCCTCCCGTAGGAGT, and 338R-II 5' GCWGCCACCCGTAGGTGT). Then, we used the QIAquick PCR Purification Kit (Qiagen, Barcelona, Spain) to purified PCR amplicons and the products were quantified by a spectrophotometer as before. After that, it was pooled in equal concentration and $2 \mathrm{nM}$ of pooled amplicons were then sequenced by Illumina MiSeq sequencer following the standard protocols.

\section{Analysis of the microbiota}

Trimmomatic was used to demultiplexed and quality-filtered the Raw data. The obtained data was merged by FLASH compliance with rules: (i) The reads attaining an average quality score less than 20 in a sliding window of $50 \mathrm{bp}$ were deleted. (ii) Primers were only allowing 2 nucleotides mismatching, meanwhile reads containing ambiguous bases were deleted. (iii) Sequences containing overlap over $10 \mathrm{bp}$ were merged through the overlap sequence.
Majorbio I-Sanger Cloud Platform was used to analyze the microbiota (https://www.i-sanger.com). We used the UPARSE to clustered the operational taxonomic units (OTUs) with a cutoff of $97 \%$ similarity (version 7.1 ) as well as UCHIME were used to identified and removed chimeric sequences. RDP Classifier was used to analyzed the taxonomy of each sequence through algorithm against the Silva (SSU128) 16S rRNA database by a confidence threshold of 70\% (http://rdp.cme.msu.edu/).

We normalized the OTUs abundance of each sample based on the least sequences number of all samples and removed the rare OTUs. Then, it was used to analyze the alpha diversity and beta diversity by QIIME tool. Meanwhile, linear discriminant analysis (LDA) effect size (LEfSe) analyses were carried out through the LEfSe tool with an LDA of 2.0. The analysis of similarity (ANOSIM) test were used to evaluate the differences of microbial structure between $\mathrm{CI}$ patients and healthy control.

\section{Biochemical assays}

Blood samples were gathered from patients with stroke at admission and from healthy subjects at physical examination center. Serum levels of low-density lipoprotein (LDL), glucose of blood (GLU), high-density lipoprotein (HDL), uric acid (UA), triglycerides (TG), and homocysteine $(\mathrm{HCY})$ were measured using standard techniques.

\section{Statistic methods}

All data are demonstrated as mean $\pm \mathrm{SD}$. The normality of the distribution had been demonstrated with Kolmogorov-Smirnoff statistic of the data. The Chi-square-tests was used to assess gender. The T-tests was used for analysis of continuous variables. Spearman's rank correlation was used to analysis of statistical dependence of continuous variables. $P$-values $<0.05$ were considered as statistically significant. Analyses were carried out through the SPSS statistical package, version 24.0.

\section{Additional files}

Additional file 1: Table S1. The serum index of $\mathrm{Cl}$ patient and healthy control. (PDF 140 kb)

Additional file 2: Table S2. The severity and outcome of $\mathrm{Cl}$ patients. (PDF 107 kb)

\section{Abbreviations}

$\mathrm{Cl}$ : Cerebral ischemic stroke; GLU: Glucose of blood; HCY: Homocysteine; HDL: High-density lipoprotein; LDL: Low-density lipoprotein;

OTU: Operational taxonomic unit; TG: Triglycerides; UA: Uric acid

\section{Acknowledgements}

Not applicable.

\section{Authors' contributions}

All authors participated in the conception and design of the study; conceived and drafted the manuscript: NL, XCW; performed the experiments: $\mathrm{NL}, \mathrm{XCW}, \mathrm{CCS}, \mathrm{XWW}, \mathrm{XJZ}, \mathrm{NW}$ and XBW; collected the basic patient 
information, clinical indicators, and imaging data: $\mathrm{ML}, \mathrm{XY}, \mathrm{TW}$, and $\mathrm{XLY}$; analyzed the data: CCS and XBW; revised the paper: CCS, XWW and XBW. All authors read and approved the final manuscript.

\section{Funding}

This research was supported by Shandong Provincial Natural Science Foundation, China (Grant NO. ZR2016HB37), the Shandong Medical Science and Technology Development Plan Project (NO. 2017WS433 and 2017WSA01044), Science and technology projects of Jinan health and family planning commission (NO. 2016-1-50).

\section{Availability of data and materials}

All data generated or analyzed during this study are included in this published article.

\section{Ethics approval and consent to participate}

The protocol of this study was approved by the local Ethical Committee of the Qilu Hospital of Shandong University (KYLL-2017(KS)-088). All volunteers received information concerning their participation in the study and gave written informed consent

\section{Consent for publication}

Not applicable.

\section{Competing interests}

The authors declare that they have no competing interests.

\section{Author details}

'Department of Dermatology, Affiliated Hospital of Shandong University of Traditional Chinese Medicine, Jinan 250011, Shandong, China. ${ }^{2}$ Department of Nephrology, Qilu Children's Hospital of Shandong University, Jinan 250022, China. ${ }^{3}$ Department of Neurology, Qilu Hospital of Shandong University, Jinan 250012, China. ${ }^{4}$ Department of Geriatric Medicine, Qilu Hospital of Shandong University; Key Laboratory of Cardiovascular Proteomics of Shandong Province, Qilu Hospital of Shandong University, Jinan 250012, China. ${ }^{5}$ Department of Neurology, Feicheng Mining Center Hospital of Shandong Province, Feicheng, Tai an 271608, China.

Received: 5 May 2019 Accepted: 24 July 2019

Published online: 19 August 2019

\section{References}

1. Hossmann KA. Pathophysiology and therapy of experimental stroke. Cell Mol Neurobiol. 2006;26(7-8):1057-83.

2. Parr E, Ferdinand P, Roffe C. Management of Acute Stroke in the Olde Person. Geriatrics. 2017:2(3):27.

3. Chamorro A, Urra X, Planas AM. Infection after acute ischemic stroke: a manifestation of brain-induced immunodepression. Stroke. 2007;38(3):1097103.

4. Zeng $X$, et al. Higher risk of stroke is correlated with increased opportunistic pathogen load and reduced levels of butyrate-producing Bacteria in the gut. Front Cell Infect Microbiol. 2019;9:4.

5. ladecola C, Anrather J. The immunology of stroke: from mechanisms to translation. Nat Med. 2011;17(7):796-808.

6. Houlden A, et al. Brain injury induces specific changes in the caeca microbiota of mice via altered autonomic activity and mucoprotein production. Brain Behav Immun. 2016:57:10-20.

7. Wen SW, Wong CHY. An unexplored brain-gut microbiota axis in stroke. Gut Microbes. 2017:8(6):601-6.

8. Stanley D, Moore RJ, Wong CHY. An insight into intestinal mucosal microbiota disruption after stroke. Sci Rep. 2018;8(1):568.

9. Benakis $C$, et al. Commensal microbiota affects ischemic stroke outcome by regulating intestinal gammadelta T cells. Nat Med. 2016;22(5):516-23.

10. Sherwin E, Dinan TG, Cryan JF. Recent developments in understanding the role of the gut microbiota in brain health and disease. Ann N Y Acad Sci. 2018:1420(1):5-25.

11. Winek $\mathrm{K}$, et al. Depletion of cultivatable gut microbiota by broad-Spectrum antibiotic pretreatment worsens outcome after murine stroke. Stroke. 2016; 47(5):1354-63.
12. Yamashiro K, et al. Gut dysbiosis is associated with metabolism and systemic inflammation in patients with ischemic stroke. PLoS One. 2017; 12(2):e0171521.

13. Yin J, et al. Dysbiosis of gut microbiota with reduced trimethylamine-Noxide level in patients with large-artery atherosclerotic stroke or transient ischemic attack. J Am Heart Assoc. 2015:4(11):e002699.

14. Ji W, et al. Analysis of intestinal microbial communities of cerebral infarction and ischemia patients based on high throughput sequencing technology and glucose and lipid metabolism. Mol Med Rep. 2017;16(4):5413-7.

15. Vital M, Howe AC, Tiedje JM. Revealing the bacterial butyrate synthesis pathways by analyzing (meta) genomic data. MBio. 2014;5(2):e00889.

16. Cani PD, de Vos WM. Next-generation beneficial microbes: the case of Akkermansia muciniphila. Front Microbiol. 2017;8:1765.

17. Zoetendal EG, et al. Victivallis vadensis gen. Nov., sp. nov., a sugarfermenting anaerobe from human faeces. Int J Syst Evol Microbiol. 2003; 53(Pt 1):211-5.

18. Cummings $\mathrm{JH}$, et al. Short chain fatty acids in human large intestine, portal, hepatic and venous blood. Gut. 1987;28(10):1221-7.

19. Koh A, et al. From dietary Fiber to host physiology: short-chain fatty acids as key bacterial metabolites. Cell. 2016;165(6):1332-45.

20. Zhai $Q$, et al. A next generation probiotic, Akkermansia muciniphila. Crit Rev Food Sci Nutr. 2018; https://doi.org/10.1080/10408398.2018.1517725. [Epub ahead of print].

21. Fang D, et al. Bifidobacterium pseudocatenulatum LI09 and Bifidobacterium catenulatum LI10 attenuate D-galactosamine-induced liver injury by modifying the gut microbiota. Sci Rep. 2017:7(1):8770.

22. Zheng $L$, et al. Microbial-derived butyrate promotes epithelial barrier function through IL-10 receptor-dependent repression of Claudin-2. J Immunol. 2017;199(8):2976-84

23. Lukovac $S$, et al. Differential modulation by Akkermansia muciniphila and Faecalibacterium prausnitzii of host peripheral lipid metabolism and histone acetylation in mouse gut organoids. MBio. 2014;5(4).

24. Hanninen $\mathrm{A}$, et al. Akkermansia muciniphila induces gut microbiota remodelling and controls islet autoimmunity in NOD mice. Gut. 2018;67(8): 1445-53.

25. Kamtchum-Tatuene J, Jickling GC. Blood biomarkers for stroke diagnosis and management. NeuroMolecular Med. 2019.

26. Deng QW, et al. Triglyceride to high-density lipoprotein cholesterol ratio predicts worse outcomes after acute ischaemic stroke. Eur J Neurol. 2017; 24(2):283-91.

27. Dieplinger $B$, et al. Prognostic value of inflammatory and cardiovascular biomarkers for prediction of 90-day all-cause mortality after acute ischemic stroke-results from the Linz stroke unit study. Clin Chem. 2017;63(6):1101-9.

28. Chen $\mathrm{XL}$, et al. Serum YKL-40, a prognostic marker in patients with largeartery atherosclerotic stroke. Acta Neurol Scand. 2017;136(2):97-102.

29. Tall AR. Plasma high density lipoproteins: therapeutic targeting and links to atherogenic inflammation. Atherosclerosis. 2018;276:39-43.

30. Prospective Studies C, et al. Blood cholesterol and vascular mortality by age, sex, and blood pressure: a meta-analysis of individual data from 61 prospective studies with 55,000 vascular deaths. Lancet. 2007:370(9602): 1829-39.

\section{Publisher's Note}

Springer Nature remains neutral with regard to jurisdictional claims in published maps and institutional affiliations.

Ready to submit your research? Choose BMC and benefit from

- fast, convenient online submission

- thorough peer review by experienced researchers in your field

- rapid publication on acceptance

- support for research data, including large and complex data types

- gold Open Access which fosters wider collaboration and increased citations

- maximum visibility for your research: over $100 \mathrm{M}$ website views per year

At BMC, research is always in progress.

Learn more biomedcentral.com/submissions 